\title{
Cerebral Venous Thrombosis in Acute Lymphoblastic Leukemia with Severe COVID-19: a Case Report
}

\author{
Ishita Manral ${ }^{1} \cdot$ Nihar Duddu $^{1} \cdot$ Neelabh Nayan $^{1}$ (D) $\cdot$ Rajan Kapoor $^{1} \cdot$ Preeti Gupta $^{2} \cdot$ Anirban Gupta $^{1}$
}

Accepted: 21 April 2021 / Published online: 30 April 2021

(C) The Author(s), under exclusive licence to Springer Nature Switzerland AG 2021

\begin{abstract}
Coronavirus disease 2019 (COVID-19) caused by severe acute respiratory syndrome coronavirus 2 (SARS-CoV-2) has brought an unprecedented upheaval in our health-care systems. Amongst the many challenges posed by the disease, increased risk of thromboembolism has presented a distinct new front for increased mortality and morbidity. While there are multiple documented evidences for the same, the exact mechanism, knowledge of groups at-risk, and mitigation strategies are evolving. We present a case of a young individual who was diagnosed with acute lymphoblastic leukemia (ALL), was started on appropriate chemotherapy, and subsequently developed severe COVID-19 pneumonia. He was treated for COVID-19 pneumonia and recovered from the illness. However, his recovery from COVID-19 was further complicated by cortical venous sinus thrombosis (CVT). Contrast enhanced magnetic resonance imaging (CEMRI) brain and magnetic resonance venography (MRV) revealed the diagnosis of CVT with hemorrhagic parenchymal changes. He was managed with therapeutic anticoagulation and cerebral decongestants and was subsequently shifted to oral anticoagulant therapy. While the case was managed at a tertiary care setting, it opened up the question of identifying the high-risk groups and to formulate guidelines for extended thromboprophylaxis in these patients.
\end{abstract}

Keywords Acute lymphoblastic leukemia $\cdot$ COVID-19 $\cdot$ Severe COVID-19 pneumonia $\cdot$ Cortical venous sinus thrombosis

\section{Introduction}

Severe acute respiratory syndrome coronavirus 2 (SARSCoV-2) causing coronavirus disease 2019 (COVID-19) has posed unique challenges to medical community, due to rapidity of spread of this virus across the globe. One of the challenges is increased risk of both venous and arterial thromboembolism associated with COVID-19 and its management (1-5). It has now been well established that at least all severe cases of COVID-19 pneumonia should be on prophylactic or therapeutic dose of anticoagulant. However, the duration of anticoagulation and requirement of post-discharge thromboprophylaxis is not clear and is largely driven by the treating physician's perspective.

This article is part of the Topical Collection on Covid-19

Neelabh Nayan

neelabh.nayan@gmail.com

1 Department of Medicine, Command Hospital, Kolkata, India

2 Department of Radiodiagnosis, Command Hospital, Kolkata, India
Herein, we report a case of acute lymphoblastic leukemia (ALL), with severe COVID-19 pneumonia, who subsequently developed cortical venous sinus thrombosis (CVT) following recovery from COVID-19.

\section{Case}

\section{Patient Information and Clinical Findings}

Twenty-seven-year-old male, who was previously in good health, presented in early May 2020 with intermittent fever, non-productive cough, subconjunctival hemorrhage (right eye), and symptomatic anemia. On clinical evaluation, he had pallor and submandibular lymphadenopathy. Consequent evaluation established a diagnosis of precursor $\mathrm{B}$ cell ALL with Philadelphia $(\mathrm{Ph})$ chromosome positivity. As per the obligatory protocol at our center, he also underwent screening for infection by SARS-CoV-2 and was negative by reverse transcriptase polymerase chain reaction (RT-PCR) for the same. He was started on Tab Dasatinib $140 \mathrm{mg}$ PO once daily along with chemotherapy as per adult GMALL protocol. 
He had, for the most part, an uneventful induction phase till day 21 of protocol when he developed fever with chills. His RT-PCR returned a positive report of SARS-CoV-2 infection, and he was shifted to the COVID isolation facility of the hospital. On third day post diagnosis, he developed cough and worsening breathlessness. His oxygen saturation dropped to $90 \%$ and he had to be shifted to intensive care unit (ICU). His chest radiograph was suggestive of COVID-19 pneumonia along with bilateral pleural effusion. He was managed with high flow nasal oxygen (HFNO) by non-invasive ventilation (NIV), Inj Dexamethasone $6 \mathrm{mg}$ once daily, Inj Enoxaparin $40 \mathrm{mg}$ twice daily, and a 5-day course of Inj Remdesivir (200 mg loading followed by $100 \mathrm{mg}$ once daily). Dasatinib was stopped and Imatinib introduced keeping the possibility of Dasatinib-induced/aggravated pleural effusion in mind. He responded to treatment and was discharged on day 14 of admission after negative RT-PCR for SARS-CoV-2. At discharge, he was advised to continue Tab Imatinib $400 \mathrm{mg}$ twice daily along with prophylactic dose of acyclovir and septran.

One week following the discharge, he developed weakness of the left hand. On evaluation in the medical ward, he was found to be confused with inability to recall recent events. The patient was reviewed by a neurologist. Mini mental state examination (MMSE) showed score of 19/30 with impairment of language, recall, attention, and calculation. No sensory, motor, or autonomic deficits were observed on the neurological examination.
Fig. 1 Images show curvilinear T2/ FLAIR hypointensities with corresponding T1 hyperintensity and significant peri-lesional edema in left frontoparietal lobes. Multiple blooming artifacts on gradient echo images confirm them as thrombosed cortical veins
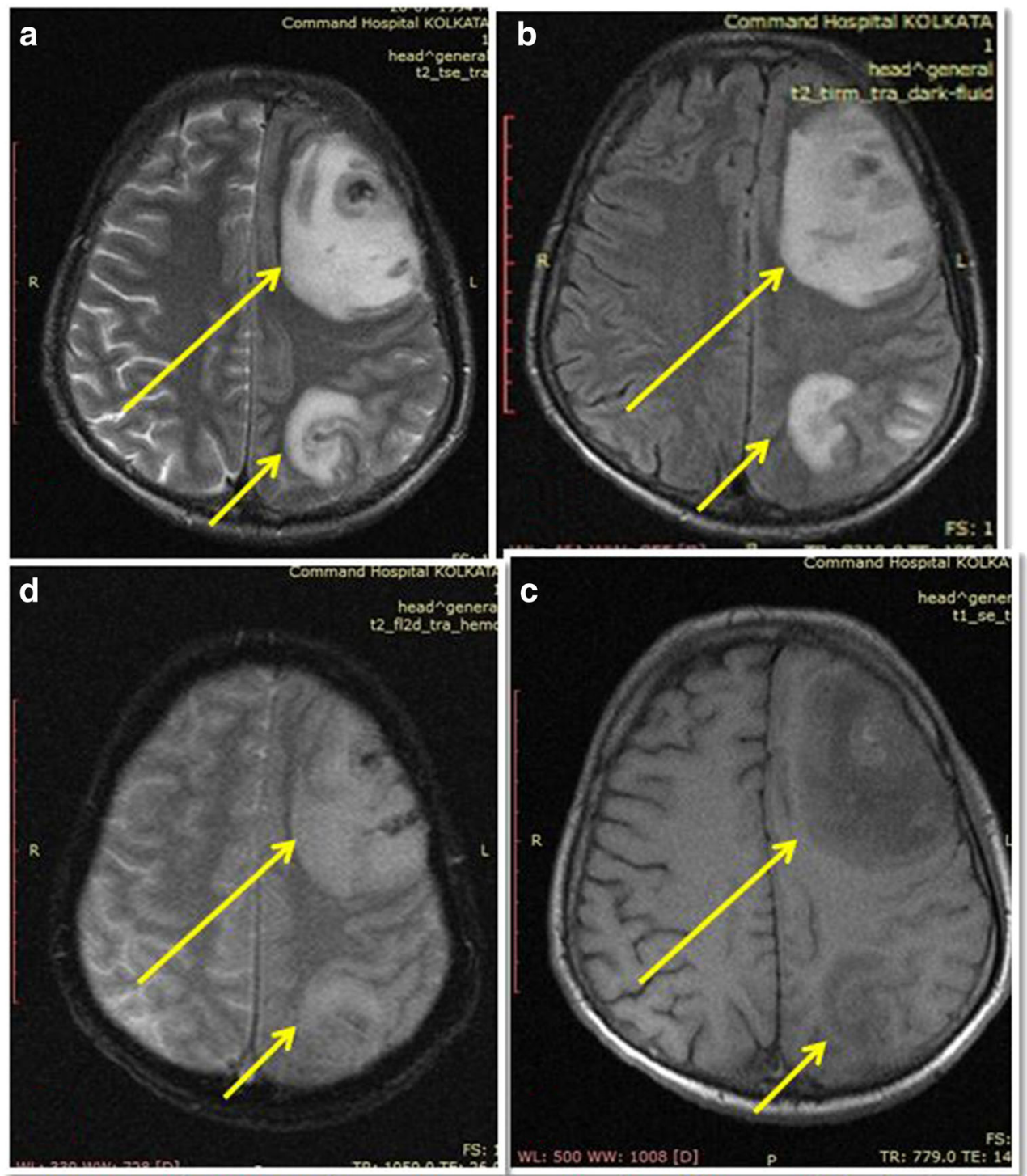

CEMRI and MRV Brain sequences, arranged as (from top left, clock-wise) a. T2, b. FLAIR, c. T1, d. GRE Axial images 


\section{Diagnostic Assessment}

As per the decision of the treating team, a contrast enhanced magnetic resonance imaging (CEMRI) brain and magnetic resonance venography (MRV) were done. It did not show any signal loss in major venous sinuses or abnormal post contrast enhancement. Multiple blooming artifacts on gradient echo images confirmed them as thrombosed cortical veins. The findings were, therefore, suggestive of cerebral venous thrombosis (CVT) with hemorrhagic parenchymal changes (Fig. 1). He was screened for other potential venous thromboembolic (VTE) events. However, electrocardiography, color Doppler of the lower limbs, chest radiograph, and 2-D echocardiography were negative for any suggestion of deep venous thrombosis (DVT) of the lower limbs or pulmonary embolism (PE).

\section{Therapeutic Intervention, Follow-up, and Outcomes}

He was subsequently managed in the medical ward with therapeutic dose of Inj Enoxaparin and cerebral decongestants. He had uneventful recovery and was shifted over to Tab Apixaban after 2 weeks. He was symptomatically better during the subsequent monthly follow-up visits at both the neurology and hematology outpatient departments, and repeat MMSE evaluations showed graded improvement during the follow-up visits. There were no adverse or unanticipated outcomes during the follow-up period of 3 months.

\section{Timeline}

The timeline of the important events is depicted in Fig. 2.

\section{Discussion}

We report an unusual case of thrombosis post-discharge in a patient of high risk ALL after recovery from COVID-19 pneumonia.

Amongst patients suffering from COVID-19, the incidence of various thrombotic events is $16.7-31 \%(4,6)$.

In the study by Klok et al. (4), out of the 184 ICU patients who were confirmed to have COVID-19 pneumonia, the cumulative incidence of various thromboembolic (TE) events was $31 \%$. Venous TE (VTE) was confirmed in $27 \%$ by the means of computed tomography pulmonary angiogram (CTPA) and/or ultrasonography, and arterial thrombotic events in 3.7\%. In March 2020, Helms et al. (6) attempted to analyze TE events between non-COVID-19 acute respiratory distress syndrome (ARDS) and COVID-19 ARDS patients. They found the presence of life-threatening thrombotic

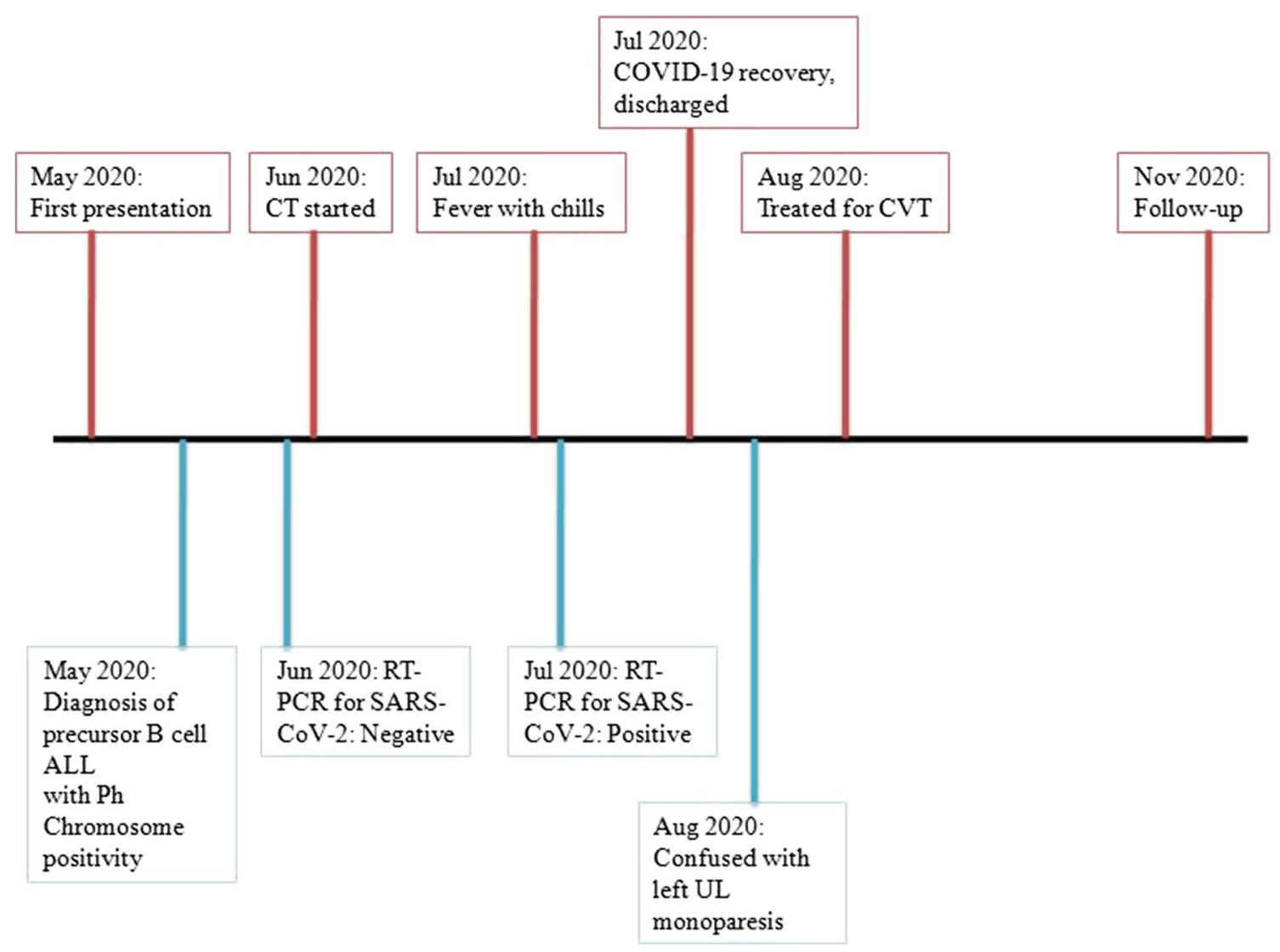

Fig. 2 A timeline of the important events of the case 
complications, mainly pulmonary embolism (PE) in $11.7 \%$, a high proportion of patients who had ARDS secondary to COVID-19. Other studies have also been done on this association and it appears that $\mathrm{PE}$ is the most common manifestation of VTE in severe COVID-19 $(7,8)$, and the risk does not appear to reduce with VTE prophylaxis $(4,9)$. Cavalcanti et al. (10) reported a case series of three patients with confirmed COVID-19 who were younger than 41 years of age and had TE complication in the form of cerebral venous thrombosis (CVT). The median time of progression from COVID-19 to CVT was 7 days in the series. Of the three, one was also diagnosed with new-onset diabetic ketoacidosis (DKA), and another was on oral contraceptive pills (OCP), both of which can independently predispose to CVT. All three had fatal outcomes from the disease.

Important risk factors for CVT include anemia, hematological disorders, and cancers, with pro-coagulopathic states and infections acting as associated factors for development of CVT. In their review of factors causing CVT, Sidhom et al. (11) found that thrombophilia, obstetric and gynecologic causes, and sepsis were the predominant precipitants. Appenzeller et al. (12) reviewed records of 24 patients with CVT over a 10-year period and found that the leading causes that could be detected included pregnancy or puerperium in $25 \%$, inherited prothrombotic state and OCP use in 17\% each, and systemic disease in $13 \%$. Comparative studies in thrombosis amongst patients report 3-6 times higher rate of thrombosis in COVID-19 patients versus non-COVID.

Liew et al. (13) propose that extended-duration thromboprophylaxis and not short-duration can possibly mitigate the risk for developing DVT and non-fatal PE in hospitalized medical patients. Patients admitted to the hospital for acute medical illness have an increased risk of VTE up to 90 days after discharge, and therefore a similar risk is possible for patients with COVID-19. However, in their recently published study, Roberts et al. (5) compared the risk of hospitalassociated VTE (HA-VTE) by comparing 42-day post-discharge outcomes of VTE in patients with COVID-19 with 18,159 discharges in 2019. They found that the odds ratio for post-discharge HA-VTE in COVID-19 was 1.6 (95\% CI, 0.77-3.1). It may be reasonable to consider extended treatment to prevent TE events after discharge from the hospitals especially in patients with procoagulant state like cancers. However, with the uncertainty over the risk-benefit of postdischarge TE prophylaxis as compared to normal medical patients, trials to evaluate the role of this are the need of the hour, more so in patients with hypercoagulable states like cancer.

\section{Conclusion}

An unusual presentation of cerebral venous thrombosis in a young patient of acute leukemia in remission, post recovery from severe COVID-19 is reported. There is an urgent need to systematically study incidence of thrombosis post recovery from COVID-19, to identify the groups that are at high risk for TE events, and to institute trials in order to create management guidelines for extended thromboprophylaxis.

Availability of Data and Material All relevant data and material is available with the corresponding author.

Code Availability Not applicable.

Author Contributions All the listed authors have made substantial contributions to the conception and the design of the study and the acquisition, analysis, or interpretation of sections of data for the work. All the listed authors have been involved in drafting the paper and revising it critically for important intellectual content, and all were involved in the final approval of the version to be published. All the authors agree to be held accountable for all aspects of the work in ensuring that questions related to the accuracy or integrity of any part of the work are appropriately investigated and resolved.

\section{Declarations}

Ethics Approval Ethics approval was waived off, in view of the ongoing pandemic and relevance of the study in mitigating the same. However, all procedures performed in studies involving human participants were in accordance with the ethical standards of the institutional and/or national research committee and with the 1964 Helsinki Declaration and its later amendments or comparable ethical standards.

Consent to Participate All the participants in the study were included after provision of informed written consent.

Consent for Publication Not applicable.

Conflict of Interest The authors declare no competing interests.

\section{References}

1. Wang T, Chen R, Liu C, Liang W, Guan W, Tang R, et al. Attention should be paid to venous thromboembolism prophylaxis in the management of COVID-19. Lancet Haematol. 2020;7(5):e362-3.

2. Tang N, Bai H, Chen X, Gong J, Li D, Sun Z. Anticoagulant treatment is associated with decreased mortality in severe coronavirus disease 2019 patients with coagulopathy. J Thromb Haemost. 2020;18(5):1094-9.

3. Patell R, Bogue T, Koshy A, Bindal P, Merrill M, Aird WC, et al. Postdischarge thrombosis and hemorrhage in patients with COVID19. Blood. 2020;136(11):1342-6.

4. Klok FA, Kruip MJHA, van der Meer NJM, Arbous MS, Gommers DAMPJ, Kant KM, et al. Incidence of thrombotic complications in critically ill ICU patients with COVID-19. Thromb Res. 2020;191: $145-7$.

5. Roberts LN, Whyte MB, Georgiou L, Giron G, Czuprynska J, Rea $\mathrm{C}$, et al. Postdischarge venous thromboembolism following hospital admission with COVID-19. Blood. 2020;136(11):1347-50.

6. Helms J, Tacquard C, Severac F, Leonard-Lorant I, Ohana M, Delabranche X, et al. High risk of thrombosis in patients with 
severe SARS-CoV-2 infection: a multicenter prospective cohort study. Intensive Care Med. 2020;46(6):1089-98.

7. Klok FA, Kruip MJHA, van der Meer NJM, Arbous MS, Gommers D, Kant KM, et al. Confirmation of the high cumulative incidence of thrombotic complications in critically ill ICU patients with COVID-19: an updated analysis. Thromb Res. 2020;191:148-50.

8. Cui S, Chen S, Li X, Liu S, Wang F. Prevalence of venous thromboembolism in patients with severe novel coronavirus pneumonia. J Thromb Haemost. 2020;18(6):1421-4.

9. Middeldorp S, Coppens M, van Haaps TF, Foppen M, Vlaar AP, Müller MCA, et al. Incidence of venous thromboembolism in hospitalized patients with COVID-19. J Thromb Haemost. 2020;18(8): 1995-2002.

10. Cavalcanti DD, Raz E, Shapiro M, Dehkharghani S, Yaghi S, Lillemoe K, et al. Cerebral venous thrombosis associated with COVID-19. Am J Neuroradiol. 2020;41(8):1370-6.
11. Sidhom Y, Mansour M, Messelmani M, Derbali H, Fekih-Mrissa $\mathrm{N}$, Zaouali J, et al. Cerebral venous thrombosis: clinical features, risk factors, and long-term outcome in a Tunisian cohort. J Stroke Cerebrovasc Dis. 2014;23(6):1291-5.

12. Appenzeller S, Zeller CB, Annichino-Bizzachi JM, Costallat LTL, Deus-Silva L, Voetsch B, et al. Cerebral venous thrombosis: influence of risk factors and imaging findings on prognosis. Clin Neurol Neurosurg. 2005;107(5):371-8.

13. Liew AYL, Piran S, Eikelboom JW, Douketis JD. Extendedduration versus short-duration pharmacological thromboprophylaxis in acutely Ill hospitalized medical patients: a systematic review and meta-analysis of randomized controlled trials. J Thromb Thrombolysis. 2017;43(3):291-301.

Publisher's Note Springer Nature remains neutral with regard to jurisdictional claims in published maps and institutional affiliations. 